\title{
COMPACT AND MAJORIZABLE FUNCTIONALS OF FINITE TYPE
}

\author{
MARC BEZEM
}

The main result of this paper will be that various notions of majorizability and compactness coincide in the full typestructure over the natural numbers. Moreover we shall show that the extensional typestructure of strongly majorizable functionals can be obtained by applying Zucker's construction ( $)^{\mathrm{E}}$ to any of these coinciding intensional typestructures. A different result is proved in the typestructure of effective operations, where not every majorizable functional is compact. Finally we shall introduce the concept of relative compactness in the full typestructure and prove that there are just two degrees of compactness.

\section{§1. Preliminaries.}

1.0. Types are 0 and with $\sigma$ and $\tau$ also $(\sigma) \tau$ (often written as $\sigma \rightarrow \tau$ in the literature). We shall use the symbols $\sigma, \tau, \rho$ to denote types. Pure types (denoted by natural numbers) are 0 and with $n$ also $n+1=(n) 0$.

A typestructure $\mathfrak{I}=U \mathfrak{I}_{\sigma}$ is given by specifying sets $\mathfrak{I}_{\sigma}$ for each type $\sigma$ and application mappings $\left(\mathfrak{I}_{(\sigma) \tau} \times \mathfrak{I}_{\sigma}\right) \rightarrow \mathfrak{I}_{\tau}$ for all $\sigma, \tau$. We shall only consider typestructures over the natural numbers, i.e. with $\mathfrak{I}_{0}=\mathbf{N}$. $\subseteq=\bigcup \mathfrak{S}_{\sigma}$ is called a subtypestructure of $\mathfrak{I}$ if $\mathfrak{\Xi}_{\sigma} \subseteq \mathfrak{I}_{\sigma}$ for all $\sigma$ and $\mathfrak{\Xi}$ is closed under application in $\mathfrak{I}$. Elements of $\mathfrak{I}_{\sigma}$ are called functionals of type $\sigma$. Functionals (as well as variables for functionals) will mostly be denoted by capitals, but whenever we wish to emphasize that a functional (or variable) is of type zero, i.e. a natural number, we shall denote it by a lower case letter. $F \in \sigma$ expresses that $F$ is a functional of type $\sigma$. Application of a functional $F \in(\sigma) \tau$ to a functional $G \in \sigma$ yields a functional of type $\tau$, which we shall denote by $F G$. In expressions like $F G H$ association will always be assumed to be to the left and the functionals will be assumed to have appropriate types. We shall occasionally use lambda-notation to specify functionals, i.e. $\lambda X$. - specifies a functional $F$ such that $F X=-$ for all $X$.

Two notions of equality for functionals can be distinguished, namely intensional and extensional equality. Let $\mathfrak{I}=\bigcup \mathfrak{I}_{\sigma}$ be a typestructure. Intensional equality of type $\sigma$ is equality on $\mathfrak{I}_{\sigma}$ as given to us. Extensional equality of type $(\sigma) \tau$ is hereditarily defined by

$$
F={ }_{(\sigma) \tau} F^{\prime} \quad \text { iff } \quad F, F^{\prime} \in \mathfrak{I}_{(\sigma) \tau} \wedge \forall X \in \mathfrak{I}_{\sigma} F X={ }_{\tau} F^{\prime} X .
$$

Received May 12, 1987; revised October 26, 1987. 
Extensional equality of type 0 is equality on $\mathfrak{I}_{0}$ as given to us. $\mathcal{I}$ is called extensional if all application mappings respect extensional equality. Otherwise $\mathcal{I}$ is called intensional.

The full typestructure over the natural numbers $\mathfrak{I}=\bigcup \mathfrak{I}_{\sigma}$ is inductively defined by $\mathfrak{I}_{0}=\mathrm{N}$ and $\mathfrak{I}_{(\sigma) \tau}=\left\{F \mid F: \mathfrak{I}_{\sigma} \rightarrow \mathfrak{I}_{\tau}\right\}$ (with set-theoretical application; note that intensional and extensional equality coincide in $\mathfrak{I}$, and so $\mathfrak{I}$ is extensional). In the first five sections as well as in the last, variables for functionals are taken to range over $\mathcal{I}$. In all other sections this range will be specified, e.g. by $\forall F \in \mathfrak{M}$ or $\exists y \in$ $\mathrm{HEO}_{2}$. Constant functionals of type $\sigma$ will be denoted by $n^{\sigma}, n \in \mathbf{N}$.

1.1. The typestructures MAJ, s-MAJ, $\omega-\mathrm{MAJ}$ and COMPACT (intensional subtypestructures of the full typestructure over $\mathbf{N}$ ) are inductively defined as follows.

MAJ:

$n$ majo $_{0} m$ iff $n \geq m$;

$F^{*} \operatorname{maj}_{(\sigma) \tau} F$ iff $\forall G^{*}, G\left(G^{*} \operatorname{maj}_{\sigma} G \rightarrow F^{*} G^{*} \operatorname{maj}_{\tau} F G\right)$.

$F \in \operatorname{MAJ}\left(F\right.$ is majorizable) iff $\exists F^{*} F^{*}$ maj $F$.

$s-\mathrm{MAJ}$ :

$n s$-majo $m$ iff $n \geq m$;

$F^{*} \mathrm{~s}-\mathrm{maj}_{(\sigma) \tau} F$ iff $\forall G^{*}, G\left(G^{*} \mathrm{~s}-\mathrm{maj}_{\sigma} G \rightarrow F^{*} G^{*} \mathrm{~s}-\mathrm{maj}_{\tau} F^{*} G, F G\right)$.

$F^{*} \mathrm{~s}-\mathrm{maj}_{(\sigma) \tau} F$ iff $\forall G^{*}, G\left(G^{*} \mathrm{~s}-\mathrm{maj}_{\sigma} G \rightarrow F^{*} G^{*} \mathrm{~s}-\mathrm{maj}_{\tau} F^{*} G, F G\right)$.

$\omega$-MAJ:

$\omega$-maj $\mathbf{j}_{\sigma}$ is an $\omega$-ary relation on $\mathfrak{I}_{\sigma}$.

$\left(n_{0}, n_{1}, \ldots\right) \in \omega-$ majoj $_{0}$ iff $n_{0} \leq n_{1} \leq \cdots$;

$\left(F_{0}, F_{1}, \ldots\right) \in \omega-\mathrm{maj}_{(\sigma) \tau}$ iff $\forall\left(G_{0}, G_{1}, \ldots\right) \in \omega-\mathrm{maj}_{\sigma}\left(F_{0} G_{0}, F_{1} G_{1}, \ldots\right) \in \omega-\mathrm{maj}_{\tau}$.

$F \in \omega$-MAJ ( $F$ is $\omega$-majorizable $)$ iff $\exists\left(F_{0}, F_{1}, \ldots\right) \in \omega$-maj $F_{0}=F$.

COMPACT:

$\mathfrak{A} \subset \mathfrak{I}_{0}$ is compact iff $\mathfrak{U}$ is finite;

$\mathfrak{B} \subset \mathfrak{I}_{(\sigma) \tau}$ is compact iff $\forall \mathfrak{C} \subset \mathfrak{I}_{\sigma}(\mathbb{C}$ is compact $\rightarrow \mathfrak{B C}=\{B C \mid B \in \mathfrak{B} \wedge C \in \mathbb{C}\}$ is compact);

$F \in$ COMPACT ( $F$ is compact) iff the set $\{F\}$ is compact.

Application and (intensional) equality in MAJ, s-MAJ, $\omega$-MAJ and COMPACT are induced by the full typestructure. Type subscripts will often be omitted.

Both the compact functionals and the majorizable functionals were introduced by Howard in [T, 2.8.6 and Appendix]. The strongly majorizable functionals were introduced as the extensional typestructure $\mathfrak{M}$ in [B2]. The relation $\omega$-maj is a modification of the relation maj, entailing the property that all majorants are in $\omega$ MAJ. This property, also enjoyed by s-maj but not by maj, is of interest in proving that a typestructure is a model of bar recursion (see [B2]). Although we failed to find an elegant proof that $\omega$-MAJ satisfies bar recursion, the relation $\omega$-maj seems to be interesting in itself.

1.2. In this paper proofs frequently use the following form of the comprehension axiom:

$\left(\mathrm{CA}_{\sigma, \tau}\right) \quad(\forall F \in \sigma \exists ! G \in \tau P(F, G)) \rightarrow \exists H \in(\sigma) \tau \forall F \in \sigma P(F, H F)$,

where $\exists$ ! means "there exists a unique". Whenever this is the case, it will be indicated by $(\mathrm{CA})$.

Strictly speaking the relations s-maj in this paper and in [B2] are different, since they are defined on different domains ( $\mathfrak{I}$ instead of $\mathfrak{M})$. However, all lemmas of 
$[\mathrm{B} 2, \S 1]$ can easily be proved to hold for the relations s-maj in this paper. Only two of these lemmas will be used here. For ease of reference we restate them in 1.3 and 1.4 below. Proofs may be gleaned from [B2].

1.3. Lemma. $F^{*}$ s-maj $F \rightarrow F^{*}$ s-maj $F^{*} \rightarrow F^{*} \in$ s-MAJ.

1.4. Lemma. For all $\sigma=\left(\sigma_{1}\right) \cdots\left(\sigma_{k}\right) \tau$ we have: $F^{*}$ s-maj $F$ iff $\forall G_{1}^{*}, G_{1}, \ldots, G_{k}^{*}$, $G_{k}\left(\left(G_{1}^{*}\right.\right.$ s-maj $G_{1} \wedge \cdots \wedge G_{k}^{*}$ s-maj $\left.G_{k}\right) \rightarrow F^{*} G_{1}^{*} \cdots G_{k}^{*}$ s-maj $F^{*} G_{1}^{*} \cdots G_{k-1}^{*} G_{k}, \ldots$, $\left.F^{*} G_{1} \cdots G_{k}, F G_{1} \cdots G_{k}\right)$.

1.5. For the remaining part of this paper we use $\vec{G}, \vec{X}$, etc. as abbreviations of finite sequences $G_{1}, \ldots, G_{k}, X_{1}, \ldots, X_{k}$, etc., of functionals of appropriate types. Furthermore $\vec{G}^{*}(\mathrm{~s}-)$ maj $\vec{G}$ abbreviates $G_{1}^{*}(\mathrm{~s}$ ) $)$ maj $G_{1} \wedge \cdots \wedge G_{k}^{*}$ (s-)maj $G_{k}$.

1.6. As observed by Kohlenbach in [K], Lemma 1.4 can be simplified by combining it with Lemma 1.3 into the following

Lemma. For all $\sigma=\left(\sigma_{1}\right) \cdots\left(\sigma_{k}\right) \tau$ we have: $F^{*}$ s-maj $F$ iff

$$
\forall \vec{G}^{*}, \vec{G}\left(\vec{G}^{*} \text { s-maj } \vec{G} \rightarrow F^{*} \vec{G}^{*} \text { s-maj } F^{*} \vec{G}, F \vec{G}\right) .
$$

\section{\$2. Basic facts about MAJ.}

2.1. Characterization Lemma. Let $F \in \sigma=\left(\sigma_{1}\right) \cdots\left(\sigma_{k}\right) 0$; then $F \in$ MAJ iff $\left\{F \vec{X} \mid \vec{X}^{*}\right.$ maj $\left.\vec{X}\right\}$ is finite for all $\vec{X}^{*}$.

PrOOF. " $\rightarrow$ ". Let $F \in$ MAJ; then $F^{*}$ maj $F$ for some $F^{*}$. Since $\vec{X}^{*}$ maj $\vec{X}$ implies $F^{*} \vec{X}^{*} \geq F \vec{X}$, it follows that the set $\left\{F \vec{X} \mid \vec{X}^{*}\right.$ maj $\left.\vec{X}\right\}$ is bounded by $F^{*} \vec{X}^{*}$ for every $\vec{X}^{*}$.

“๘”. (CA) Suppose $\left\{F \vec{X} \mid \vec{X}^{*}\right.$ maj $\left.\vec{X}\right\}$ is finite for every $\vec{X}^{*}$. Define $\hat{F}$ by $\hat{F} \vec{X}^{*}=$ $\max \left\{F \vec{X} \mid \vec{X}^{*}\right.$ maj $\left.\vec{X}\right\}$. Note that $\vec{X}^{*}$ maj $\overrightarrow{0}$ for all $\vec{X}^{*}$. It follows by the definition of the relation maj that $\hat{F}$ maj $F$, so $F \in$ MAJ.

2.2. Lemma. (i) Every $F \in \mathrm{MAJ}$ has a minimal majorant; in symbols:

$$
\forall F \in \operatorname{MAJ} \exists \hat{F} \text { maj } F \forall F^{*} \text { maj } F F^{*} \text { maj } \hat{F} \text {. }
$$

(ii) The relation maj is transitive.

Proof. (CA) By simultaneous induction on the type.

$\sigma=0$ : Obvious.

$(\sigma) \tau$ : Suppose the lemma has been proved for all subtypes of $\sigma$ and $\tau$.

Ad (i), let $F \in$ MAJ be of type $(\sigma) \tau=\left(\sigma_{1}\right) \cdots\left(\sigma_{k}\right) 0$. Define $\hat{F}$ as above in 2.1. Let $F^{*}$ maj $F$ and $\vec{X}^{*}$ maj $\vec{X}$. In order to obtain $F^{*}$ maj $\hat{F}$, we only have to show that $F^{*} \vec{X}^{*} \geq \hat{F} \vec{X}=\max \{F \vec{Y} \mid \vec{X}$ maj $\vec{Y}\}$. This follows from the induction hypothesis (ii), since $\vec{X}^{*}$ maj $\vec{X}$ maj $\vec{Y}$ implies $\vec{X}^{*}$ maj $\vec{Y}$ by transitivity, and hence $F^{*} \vec{X}^{*} \geq F \vec{Y}$ for all $\vec{Y}$ with $\vec{X}$ maj $\vec{Y}$.

Ad (ii), let $D, E$ and $F$ be functionals of type $(\sigma) \tau$ such that $D$ maj $E$ maj $F$. Let $G$ and $H$ be functionals of type $\sigma$ with $G$ maj $H$, hence $H \in$ MAJ. It follows by the induction hypothesis (i) that $H$ has a minimal majorant $\hat{H}$. From $G$ maj $\hat{H}$ maj $H$ follows $D G$ maj $E \hat{H}$ maj $F H$, and hence $D G$ maj $F H$ by the induction hypothesis (ii) and transitivity. It follows that $D$ maj $F$.

\section{§3. Basic facts about s-MAJ.}

3.1. LemMA. The relation s-maj is transitive.

Proof. By induction on the type.

$\sigma=0$ : Obvious. 
$(\sigma) \tau$ : Suppose the lemma has been proved for the types $\sigma$ and $\tau$. Let $D, E$ and $F$ be functionals of type $(\sigma) \tau$ such that $D$ s-maj $E$ s-maj $F$. Let $G$ and $H$ be functionals of type $\sigma$ with $G$ s-maj $H$. It follows by the definition of s-maj that $D G$ s-maj $D H$. Moreover we have $G$ s-maj $G$ by Lemma 1.3. It follows that $D G$ s-maj $E G$ s-maj $F H$ and hence, by the induction hypothesis for $\tau, D G$ s-maj $F H$. So we have $D$ s-maj $F$ by the definition of s-maj.

3.2. Characterization Lemma. Let $F \in \sigma=\left(\sigma_{1}\right) \cdots\left(\sigma_{k}\right) 0$; then $F \in \mathrm{s}-\mathrm{MAJ}$ iff $\left\{F \vec{X} \mid \vec{X}^{*}\right.$ s-maj $\left.\vec{X}\right\}$ is finite for all $\vec{X}^{*}$.

PROOF. " $\rightarrow$ " is identical to " $\rightarrow$ " in the proof of Lemma 2.1 .

“ $\leftarrow$ ". (CA) Define $\hat{F}$ by $\hat{F} \vec{X} *=\max \left\{F \vec{X} \mid \vec{X}^{*}\right.$ s-maj $\left.\vec{X}\right\}$, taking $\max \varnothing=0$. For all $\vec{X}^{*}$ s-maj $\vec{X}$ we have $\vec{F} \vec{X}^{*} \geq F \vec{X}$ by the definition of $F$, whereas $\hat{F} \vec{X}^{*} \geq \hat{F} \vec{X}$ uses the transitivity of s-maj as well. It follows by Lemma 1.6 that $\hat{F}$ s-maj $F$.

3.3. LEMMA. Every $F \in \mathrm{S}-\mathrm{MAJ}$ has a minimal s-majorant.

Proof. Let $F \in \mathrm{s}-\mathrm{MAJ}$ and define $\hat{F}$ as in 3.2. Using transitivity one proves, similar to the proof of $2.2(\mathrm{i})$, that $\hat{F}$ is a minimal s-majorant of $F$.

\section{§4. Basic facts about $\omega-\mathrm{MAJ}$.}

4.1. Lemma. If $\left(F_{0}, F_{1}, \ldots\right) \in \omega-\mathrm{maj}_{\sigma}$, then $\left(F_{k}, F_{k+1}, \ldots\right) \in \omega-\mathrm{maj}_{\sigma}$ and $\left(0^{\sigma}, \ldots, 0^{\sigma}\right.$, $\left.F_{0}, F_{1}, \ldots\right) \in \omega-$ maj $_{\sigma}\left(k\right.$ times $\left.0^{\sigma}\right)$ for every $k$.

Proof. By simultaneous induction on the type.

$\sigma=0$ : Obvious.

$(\sigma) \tau$ : Suppose the lemma has been proved for the types $\sigma$ and $\tau$. Let $\left(F_{0}, F_{1}, \ldots\right)$ $\in \omega-\operatorname{maj}_{(\sigma) \tau}$ and $\left(X_{0}, X_{1}, \ldots\right) \in \omega-$ maj $_{\sigma}$. Then we have by the induction hypothesis $\left(0^{\sigma}, \ldots, 0^{\sigma}, X_{0}, X_{1}, \ldots\right) \in \omega-\mathrm{maj}_{\sigma}$ and $\left(X_{k}, X_{k+1}, \ldots\right) \in \omega-\mathrm{maj}_{\sigma}$ for every $k$. Hence

$$
\left(F_{0} 0^{\sigma}, \ldots, F_{k-1} 0^{\sigma}, F_{k} X_{0}, F_{k+1} X_{1}, \ldots\right) \in \omega-\mathrm{maj}_{\tau}
$$

and $\left(F_{0} X_{k}, F_{1} X_{k+1}, \ldots\right) \in \omega-\mathrm{maj}_{\tau}$. Again by the induction hypothesis it follows that $\left(F_{k} X_{0}, F_{k+1} X_{1}, \ldots\right) \in \omega-\mathrm{maj}_{\tau}$ and

$$
\left(0^{(\sigma) \tau} X_{0}, \ldots, 0^{(\sigma) \tau} X_{k-1}, F_{0} X_{k}, F_{1} X_{k+1}, \ldots\right) \in \omega-\mathrm{maj}_{\tau} .
$$

Hence $\left(F_{k}, F_{k+1}, \ldots\right) \in \omega-\mathrm{maj}_{(\sigma) \tau}$ and $\left(0^{(\sigma) \tau}, \ldots, 0^{(\sigma) \tau}, F_{0}, F_{1}, \ldots\right) \in \omega-\mathrm{maj}_{(\sigma) \tau}$ by the definition of $\omega$-maj.

4.2. Lemma. If $\left(F_{0}, \ldots, F_{k}, F_{k+1}, \ldots\right) \in \omega-\mathrm{maj}_{\sigma}$ and $\left(F_{k}, G_{1}, G_{2}, \ldots\right) \in \omega-\mathrm{maj}_{\sigma}$, then

$$
\left(F_{0}, \ldots, F_{k}, G_{1}, G_{2}, \ldots\right) \in \omega-\mathrm{maj}_{\sigma} .
$$

PrOOF. By induction on the type.

$\sigma=0$ : Obvious.

$(\sigma) \tau$ : Suppose the lemma has been proved for types $\sigma$ and $\tau$. Let $\left(F_{0}, \ldots, F_{k}, F_{k+1}, \ldots\right) \in \omega-$ maj $_{(\sigma) \tau}$ and $\left(F_{k}, G_{1}, G_{2}, \ldots\right) \in \omega$-maj ${ }_{(\sigma) \tau}$. We have to show that

$$
\left(F_{0} X_{0}, \ldots, F_{k} X_{k}, G_{1} X_{k+1}, G_{2} X_{k+2}, \ldots\right) \in \omega-\mathrm{maj}_{\tau}
$$

for all $\left(X_{0}, X_{1}, \ldots\right) \in \omega-\mathrm{maj}_{\sigma}$. Suppose $\left(X_{0}, X_{1}, \ldots\right) \in \omega-\mathrm{maj}_{\sigma}$; then we have $\left(F_{0} X_{0}, F_{1} X_{1}, \ldots\right) \in \omega-$ maj $_{\tau}$ by the definition of $\omega$-maj. Moreover we have $\left(X_{k}, X_{k+1}, \ldots\right) \in \omega-\mathrm{maj}_{\sigma}$ by Lemma 4.1 , and so

$$
\left(F_{k} X_{k}, G_{1} X_{k+1}, G_{2} X_{k+2}, \ldots\right) \in \omega-\mathrm{maj}_{\tau} .
$$


Hence $\left(F_{0} X_{0}, \ldots, F_{k} X_{k}, G_{1} X_{k+1}, G_{2} X_{k+2}, \ldots\right) \in \omega-$ maj $_{\tau}$ by induction.

4.3. The relation $\leq$ on $\omega-\mathrm{MAJ}_{\sigma}$ is defined as follows: $G \leq H$ ( $H$ is an $\omega$-majorant of $G$ ) iff

$$
\exists\left(F_{0}, F_{1}, \ldots\right) \in \omega-\operatorname{maj}_{\sigma} \exists i, j\left(i<j \wedge G=F_{i} \wedge H=F_{j}\right) .
$$

LEMMA. The relation $\leq$ is transitive.

PROOF. By Lemmas 4.1 and 4.2 .

4.4. Lemma. (i) If $\left(F_{0}, F_{1}, \ldots\right) \in \omega-\mathrm{maj}_{\sigma}$, then $\left(F_{0}, F_{k}, F_{k+1}, \ldots\right) \in \omega-\mathrm{maj}_{\sigma}$ for every $k>0$.

(ii) Every $F \in \omega$-MAJ has a $\leq$-minimal $\omega$-majorant. More precisely:

$$
\begin{aligned}
\forall F \in \omega-\operatorname{MAJ} \exists F \in \omega & -\operatorname{MAJ}\left((F, \hat{F}, \hat{F}, \ldots) \in \omega-\mathrm{maj}_{\sigma}\right. \\
& \left.\wedge \forall\left(F_{0}, F_{1}, \ldots\right) \in \omega-\operatorname{maj}_{\sigma}\left(F_{0}=F \rightarrow\left(\hat{F}, F_{1}, F_{2}, \ldots\right) \in \omega-\mathrm{maj}_{\sigma}\right)\right) .
\end{aligned}
$$

Proof. (CA) By simultaneous induction on the type.

$\sigma=0$ : Obvious.

$(\sigma) \tau$ : Suppose the lemma has been proved for all subtypes of $\sigma$ and $\tau$.

Ad (i), let $\left(F_{0}, F_{1}, \ldots\right) \in \omega-\mathrm{maj}_{(\sigma) \tau}$ and $\left(X_{0}, X_{1}, \ldots\right) \in \omega-\mathrm{maj}_{\sigma}$. By the induction hypothesis there exists $\hat{X}_{0}$ such that $\left(X_{0}, \hat{X}_{0}, \hat{X}_{0}, \ldots\right) \in \omega$-maj $_{\sigma}$ and $\left(\hat{X}_{0}, X_{1}, X_{2}, \ldots\right)$ $\in \omega$-maj ${ }_{\sigma}$. By Lemma 4.2 it follows that $\left(X_{0}, \hat{X}_{0}, \ldots, \hat{X}_{0}, X_{1}, X_{2}, \ldots\right) \in \omega$-maj ${ }_{\sigma}(k-1$ times $\hat{X}_{0}$ ). By the definition of $\omega$-maj it follows that

$$
\left(F_{0} X_{0}, F_{1} \hat{X}_{0}, \ldots, F_{k-1} \hat{X}_{0}, F_{k} X_{1}, F_{k+1} X_{2}, \ldots\right) \in \omega-\mathrm{maj}_{\tau},
$$

so by the induction hypothesis (i) we have $\left(F_{0} X_{0}, F_{k} X_{1}, F_{k+1} X_{2}, \ldots\right) \in \omega$-maj $_{\tau}$. Hence we have $\left(F_{0}, F_{k}, F_{k+1}, \ldots\right) \in \omega-$ maj $_{(\sigma) \tau}$.

Ad (ii). Let $F \in \omega$-MAJ; then $F=F_{0}$ for some $\left(F_{0}, F_{1}, \ldots\right) \in \omega$-maj $j_{(\sigma) \tau}$. Define $\hat{F}$ by $\hat{F} \vec{X}=\max \{F \vec{Y} \mid \vec{Y} \leq \vec{X}\}$, taking $\max \varnothing=0$, where $\vec{Y} \leq \vec{X}$ abbreviates $Y_{1} \leq X_{1} \wedge \cdots \wedge Y_{k} \leq X_{k}$. By the definition of $\leq$, the induction hypothesis (i) and Lemma 4.1 we have $Y_{i} \leq X_{i}$ iff $\exists\left(Z_{0}, Z_{1}, \ldots\right) \in \omega$-maj $\left(Y_{i}=Z_{0} \wedge X_{i}=Z_{1}\right)$ for all $1 \leq i \leq k$. Hence we have $\vec{Y} \leq \vec{X} \rightarrow F \vec{Y} \leq F_{1} \vec{X}$ (where the last inequality is of type 0 ), so the set $\{F \vec{Y} \mid \vec{Y} \leq \vec{X}\}$ is bounded for all $\vec{X}$. It follows that $\hat{F}$ is well-defined. Using the transitivity we can easily prove that $(F, \hat{F}, \hat{F}, \ldots) \in \omega-\operatorname{maj}_{(\sigma) \tau}$. Moreover, $\left\{\hat{F}, G_{1}, G_{2}, \ldots\right) \in \omega-\operatorname{maj}_{(\sigma) \tau}$ for all $\left(G_{0}, G_{1}, \ldots\right) \in \omega-\operatorname{maj}_{(\sigma) \tau}$ with $G_{0}=F$.

COROLlaRY. If $F \leq F^{*}$ and $X \leq X^{*}$, then $F X \leq F^{*} X^{*}$.

Proof. By Lemmas 4.1 and 4.4(i).

4.5. As a corollary of the proof of Lemma 4.4 we also obtain the

Characterization Lemma. Let $F \in \sigma=\left(\sigma_{1}\right) \cdots\left(\sigma_{k}\right) 0$; then $F \in \omega$-MAJ iff $\{F \vec{Y} \mid \vec{Y} \leq \vec{X}\}$ is finite for all $\vec{X}$.

4.6. REMARKS. (i) The lemmas above could create the impression that all evident properties of (weakly) increasing sequences of natural numbers generalize to higher types. This, however, is not the case. For example, for nonmonotonic $F_{0}$ it is not true that if $\left(F_{0}, F_{1}, \ldots\right) \in \omega$-maj ${ }_{1}$ then $\left(F_{0}, \ldots, F_{0}, F_{1}, \ldots\right) \in \omega$-maj $_{1}$.

(ii) One might expect that the relations maj, s-maj and $\omega$-maj can be defined in terms of each other. However, we have failed to find any positive result on this point. 
The following equivalences do not hold in general, and will subsequently be disproved for the lowest possible type.

(1) $F^{*}$ s-maj $F$ iff $F \leq F^{*}$.

(2) $F^{*}$ s-maj $F$ iff $F^{*}$ maj $F$.

(3) $F^{*}$ maj $F$ iff $F \leq F^{*}$ (cf. Corollary 4.4 ).

(4) $\left(F_{0}, F_{1}, \ldots\right) \in \omega$-maj iff $\forall k F_{k+1}$ maj $F_{k}$.

(5) $F^{*}$ s-maj $F$ iff $\left(F, F^{*}, F^{*}, \ldots\right) \in \omega$-maj.

(6) $F^{*}$ s-maj $F$ iff $F^{*}$ maj $F^{*} \wedge F^{*}$ maj $F$.

Ad (1) and (2). Take $F=0^{1}$ and $F^{*}$ any nonmonotonic function.

Ad (3), take $F=0^{2}$ and define $F^{*} \in 2$ by $F^{*} X=0$ if $X=0^{1}$ and $m$ else, where $m$ is the smallest natural number such that $X m \neq 0$. Then we have $F^{*}$ maj $F$, but not $F \leq F^{*}$, since $F^{*}$ is not $\omega$-majorizable. For, if we define functionals $D_{k} \in 1$ by $D_{k} x=1$ if $x=k$ and 0 else, then $F^{*} D_{k}=k$ for all $k \in \mathbf{N}$. Since $D_{k} \leq 1^{1}$, it follows by Corollary 4.4 for any $G$ with $F^{*} \leq G$ that $k=F^{*} D_{k} \leq G 1^{1}$ for all $k$, which is impossible.

Ad (4). Let $F^{*}$ be the functional as defined above in the disproof of (3). Define $Y \in 3$ by $Y Z=0$ if $Z=F^{*}$, and 1 otherwise. Then we have $(Y, Y, \ldots) \in \omega$-maj, but not $Y$ maj $Y$.

Ad (5) and (6), take $F=0^{2}$ and define $F^{*} \in 2$ by $F^{*} X=0$ if $X=D_{0}$, and 1 otherwise. Then we have $F^{*}$ s-maj $F$, since $X^{*}$ s-maj $X$ implies $X^{*} \neq D_{0}$ (s-majorants are monotonic). However, we do not have $F^{*}$ maj $F^{*}$ (resp. $\left(F, F^{*}, F^{*}, \ldots\right) \in \omega$-maj), since $D_{0}$ maj $0^{1}$ (resp. $\left(0^{1}, 0^{1}, D_{0}, 1^{1}, 1^{1}, \ldots\right) \in \omega$-maj).

(iii) The negative results above, and especially the nonconstructive nature of the counterexamples to (3)-(6), lead us to posing the following

Open Questions. Do there exist interrelations between maj, s-maj and $\omega$-maj? Does there exist an interesting class of functionals (e.g. the primitive recursive functionals; see [T, p. 457]) for which one or more of the equivalences (3)-(6) hold?

\$5. Main result. In this section we shall prove the main result of this paper:

$$
\text { COMPACT }=\text { MAJ }=s-M A J=\omega-\text { MAJ. }
$$

This result is an immediate consequence of the following

THEOREM. $\mathfrak{U}$ is compact iff $\exists F \forall A \in \mathfrak{U} F$ maj $A$ (resp. $F$ s-maj $A, A \leq F$ ).

Proof. (CA) By induction on the type. $\sigma=0$ : Obvious.

$(\sigma) \tau$ : Suppose the theorem has been proved for all subtypes of $\sigma$ and $\tau$. Let $(\sigma) \tau=\left(\sigma_{1}\right) \cdots\left(\sigma_{k}\right) 0$.

" $\leftarrow$ ". We take the case $\leq$. The other cases are similar. Let $\mathfrak{A}$ be such that $\forall A \in \mathfrak{A} A \leq F$ for some $F$. Let $\mathfrak{B}_{1}, \ldots, \mathfrak{B}_{k}$ be compact sets of appropriate types. By the induction hypothesis there are $X_{1}, \ldots, X_{k}$ such that $\forall B \in \mathfrak{B}_{i} B \leq X_{i}$ for all $1 \leq i$ $\leq k$. It follows by Corollary 4.4 that $\mathfrak{Q} \vec{B}$ is bounded by $F \vec{X}$. Hence $\mathfrak{A}$ is compact. " $\rightarrow$ ". Let $\mathfrak{A}$ be compact. As to maj, define $F$ by $F \vec{X}=\max \{A \vec{Y} \mid A \in \mathscr{U}$ $\wedge \vec{X}$ maj $\vec{Y}\} . F$ is well-defined since for all $\vec{X}$ the sets $\mathfrak{B}_{i}=\left\{Y_{i} \mid X_{i}\right.$ maj $\left.Y_{i}\right\}$, $1 \leq i \leq k$, are compact by the induction hypothesis, and so $\mathfrak{A} \mathfrak{B}$ is finite. It follows by the definition of $F$ that $F$ maj $A$ for all $A \in \mathfrak{A}$. As to s-maj, define $F$ by $F \vec{X}$ $=\max \{A \vec{Y} \mid A \in \mathfrak{A} \wedge \vec{X}$ s-maj $\vec{Y}\}$, taking $\max \varnothing=0$. F is well-defined for similar 
reasons as above. We have $F$ s-maj $F$ by the transitivity of s-maj, and so $F$ s-maj $A$ for all $A \in \mathfrak{U}$ by the definition of $F$. As to $\leq$, define $F$ by $F \vec{X}=\max \{A \vec{Y} \mid A$ $\in \mathfrak{A} \wedge \vec{Y} \leq \vec{X}\}$, taking $\max \varnothing=0$; then $F$ is again well-defined. By the transitivity of $\leq$ and the definition of $F$ we have $(A, F, F, \ldots) \in \omega$-maj, so $A \leq F$ for all $A \in \mathfrak{U}$.

§6. Comparison of s-MAJ and $\mathfrak{M}$.

6.1. In [B2] the extensional typestructure $\mathfrak{M}$ of strongly majorizable functionals was introduced. In this section we shall show that $\mathfrak{M}$ is isomorphic to s-MAJ' ${ }^{\mathrm{E}}$. The extensional typestructure $\mathrm{s}-\mathrm{MAJ} \mathrm{E}^{\mathrm{E}}$ is constructed from the intensional typestructure s-MAJ by applying Zucker's construction ( $)^{\mathrm{E}}$ from $[\mathrm{Z}]$. Here we shall only state the definition and a lemma, which can be proved in a similar way as Lemma 1.4 of [B1]. With the help of this lemma one can easily prove by induction that $\approx_{\sigma}$ as defined below coincides with extensional equality on s-MAJ $\mathrm{J}_{\sigma}^{\mathrm{E}}$ as defined in 1.0. Then it immediately follows from the definition that $s-\mathrm{MAJ}^{\mathrm{E}}$ is an extensional subtypestructure of the full typestructure over $\mathbf{N}$.

By induction we define relations $\approx_{\sigma} \subset \mathrm{s}-\mathrm{MAJ}_{\sigma} \times \mathrm{s}-\mathrm{MAJ}_{\sigma}$ as follows:

$$
\begin{gathered}
n \approx_{0} n^{\prime} \quad \text { iff } n=n^{\prime} ; \\
F \approx_{(\sigma) \tau} F^{\prime} \quad \text { iff } \quad F, F^{\prime} \in \operatorname{s}-M^{\prime} \mathrm{JJ}_{(\sigma) \tau} \wedge \forall G, G^{\prime}\left(G \approx_{\sigma} G^{\prime} \rightarrow F G \approx_{\imath} F^{\prime} G^{\prime}\right) .
\end{gathered}
$$

The typestructure s-MAJ ${ }^{\mathrm{E}}$ is defined by

$$
\mathrm{s}-\mathrm{MAJ}_{\sigma}^{\mathrm{E}}=\left\{F \in \mathrm{s}-\mathrm{MAJ}_{\sigma} \mid F \approx_{\sigma} F\right\} \quad \text { for all types } \sigma .
$$

Lemma. For all types $\sigma$ :

(i) the relation $\approx_{\sigma}$ is symmetric and transitive; and

(ii) for every $F \in \mathrm{s}$-maj: if $\exists F^{\prime} F \approx_{\sigma} F^{\prime}$, then $F \approx_{\sigma} F$ and so $F \in \mathrm{s}-\mathrm{MAJ}^{\mathrm{E}}$.

As stressed before, the relations s-maj are different in s-MAJ and $\mathfrak{M}$, since they are defined on different sets. It will always be clear from the context which one is meant, and the properties we use (Lemmas 1.3 and 1.6) are shared by both.

6.2. The result $\mathrm{s}-\mathrm{MAJ}^{\mathrm{E}} \cong \mathfrak{M}$ follows from the following

THEOREM. For all types $\sigma$ there exist $\Phi_{\sigma}: \mathrm{s}-\mathrm{MAJ}_{\sigma} \rightarrow \mathfrak{M}_{\sigma}$ and $\Psi_{\sigma}: \mathfrak{M}_{\sigma} \rightarrow \mathrm{s}-\mathrm{MAJ}_{\sigma}$ such that (omitting type subscripts):

(i) $\Phi(F) \Phi(G)=\Phi(F G)$ for all $F, G \in \mathrm{s}-\mathrm{MAJ}^{\mathrm{E}}$;

(ii) $\Psi(A) \Psi(B)=\Psi(A B)$ for all $A, B \in \mathfrak{M}$;

(iii) $\Psi(\Phi(F)) \approx F$ for all $F \in \mathrm{s}-\mathrm{MAJ}^{\mathrm{E}}$;

(iv) $\Phi(\Psi(A))=A$ for all $A \in \mathfrak{M}$;

(v) $F \approx F^{\prime} \rightarrow \Phi(F)=\Phi\left(F^{\prime}\right)$ for all $F, F^{\prime} \in \mathrm{s}-\mathrm{MAJ}$;

(vi) $F^{*}$ s-maj $F \rightarrow \Phi\left(F^{*}\right)$ s-maj $\Phi(F)$ for all $F^{*}, F \in$ s-MAJ;

(vii) $A^{*}$ s-maj $A \rightarrow \Psi\left(A^{*}\right)$ s-maj $\Psi(A)$ for all $A^{*}, A \in \mathfrak{M}$.

ProOF. By simultaneous induction on the type.

$\sigma=0$ : Obvious.

$(\sigma) \tau$ : Suppose the theorem has been proved for types $\sigma$ and $\tau$. This induction hypothesis is referred to by IH.

Define $\Phi_{(\sigma) \tau}(F)=\lambda X . \Phi_{\tau}\left(F \Psi_{\sigma}(X)\right)$. We have to show that $\Phi_{(\sigma) \tau}: \mathrm{s}-\mathrm{MAJ}_{(\sigma) \tau} \rightarrow \mathfrak{M}_{(\sigma) \tau}$. Let $F \in \mathrm{s}-\mathrm{MAJ}_{(\sigma) \tau}$; then we have by $\mathrm{IH}$, for all $B$,

$$
B \in \mathfrak{M}_{\sigma} \rightarrow \Psi_{\sigma}(B) \in \mathrm{s}-\mathrm{MAJ}_{\sigma}^{\mathrm{E}} \rightarrow F \Psi_{\sigma}(B) \in \mathrm{s}-\mathrm{MAJ}_{\tau} \rightarrow \Phi_{\tau}\left(F \Psi_{\sigma}(B)\right) \in \mathfrak{M}_{\tau} .
$$


Hence $\Phi_{(\sigma) \tau}(F): \mathfrak{M}_{\sigma} \rightarrow \mathfrak{M}_{\tau}$.

From $F \in \mathrm{s}^{-\mathrm{MAJ}_{(\sigma) \tau}}$ it follows that there exists $F^{*}$ such that $F^{*}$ s-maj $F$, which implies $F^{*} \in \mathrm{s}-\mathrm{MAJ}_{(\sigma) \tau}$ by Lemma 1.3 , so also $\Phi_{(\sigma) \tau}\left(F^{*}\right): \mathfrak{M}_{\sigma} \rightarrow \mathfrak{M}_{\tau}$. By IH we have for all $B, B^{*} \in \mathfrak{M}_{\sigma}$ :

$$
\begin{aligned}
B^{*} \text { s-maj } B & \rightarrow \Psi_{\sigma}\left(B^{*}\right) \text { s-maj } \Psi_{\sigma}(B) \\
& \rightarrow F^{*} \Psi_{\sigma}\left(B^{*}\right) \text { s-maj } F^{*} \Psi_{\sigma}(B), F \Psi_{\sigma}(B) \\
& \rightarrow \Phi_{\tau}\left(F^{*} \Psi_{\sigma}\left(B^{*}\right)\right) \text { s-maj } \Phi_{\tau}\left(F^{*} \Psi_{\sigma}(B)\right), \Phi_{\tau}\left(F \Psi_{\sigma}(B)\right) \\
& \rightarrow \Phi_{(\sigma) \tau}\left(F^{*}\right) B^{*} \text { s-maj } \Phi_{(\sigma) \tau}\left(F^{*}\right) B, \Phi_{(\sigma) \tau}(F) B .
\end{aligned}
$$

Hence $\Phi_{(\sigma) \tau}\left(F^{*}\right)$ s-maj $\Phi_{(\sigma) \tau}(F)$. It follows that $\Phi_{(\sigma) \tau}(F) \in \mathfrak{M}_{(\sigma) \tau}$. Note that we also proved (vi).

Define $\Psi_{(\sigma) \tau}(A)$ by $\Psi_{(\sigma) \tau}(A) Y=\Psi_{\tau}\left(A \Phi_{\sigma}(Y)\right)$ if $Y \in \mathrm{s}-\mathrm{MAJ}$ and $0^{\tau}$ otherwise. We have to show that $\Psi_{(\sigma) \tau}: \mathfrak{M}_{(\sigma) \tau} \rightarrow \mathrm{s}-\mathrm{MAJ}_{(\sigma) \tau}^{\mathrm{E}}$. Let $A \in \mathfrak{M}_{(\sigma) \tau}$; then $\Psi_{(\sigma) \tau}(A)$ clearly is a functional of the full typestructure. From $A \in \mathfrak{M}_{(\sigma) \tau}$ it follows that there exists $A^{*}$ such that $A^{*} s$-maj $A$, which implies $A^{*} \in \mathfrak{M}_{(\sigma) \tau}$. For all functionals $G^{*}, G$ of the full typestructure we have $G^{*}$ s-maj $G \rightarrow G^{*}, G \in \mathrm{s}$-MAJ by Lemma 1.3. Hence we have by IH

$$
\begin{aligned}
G^{*} \text { s-maj } G & \rightarrow \Phi_{\sigma}\left(G^{*}\right) \text { s-maj } \Phi_{\sigma}(G) \\
& \rightarrow A^{*} \Phi_{\sigma}\left(G^{*}\right) \text { s-maj } A^{*} \Phi_{\sigma}(G), A \Phi_{\sigma}(G) \\
& \rightarrow \Psi_{\tau}\left(A^{*} \Phi_{\sigma}\left(G^{*}\right)\right) \text { s-maj } \Psi_{\tau}\left(A^{*} \Phi_{\sigma}(G)\right), \Psi_{\tau}\left(A \Phi_{\sigma}(G)\right) \\
& \rightarrow \Psi_{(\sigma) \tau}\left(A^{*}\right) G^{*} \text { s-maj } \Psi_{(\sigma) \tau}\left(A^{*}\right) G, \Psi_{(\sigma) \tau}(A) G \text { for all } G^{*}, G
\end{aligned}
$$

Hence $\Psi_{(\sigma) \tau}\left(A^{*}\right)$ s-maj $\Psi_{(\sigma) \tau}(A)$. It follows that $\Psi_{(\sigma) \tau}(A) \in \mathrm{s}$-MAJ. Note that we also proved (vii).

Now we shall prove $\Psi_{(\sigma) \tau}(A) \in \mathrm{s}-\mathrm{MAJ}^{\mathrm{E}}$, which comes down to showing $\Psi_{(\sigma) \tau}(A)$ $\approx \Psi_{(\sigma) \tau}(A)$. Let $G, G^{\prime} \in \mathrm{s}-\mathrm{MAJ}_{\sigma}$; then by $\mathrm{IH}$ we have

$$
\begin{aligned}
G \approx G^{\prime} & \rightarrow \Phi_{\sigma}(G)=\Phi_{\sigma}\left(G^{\prime}\right) \in \mathfrak{M}_{\sigma} \\
& \rightarrow \Psi_{\tau}\left(A \Phi_{\sigma}(G)\right)=\Psi_{\tau}\left(A \Phi_{\sigma}\left(G^{\prime}\right)\right) \in \mathrm{s}-\mathrm{MAJ}_{\sigma}^{\mathrm{E}} \\
& \rightarrow \Psi_{\tau}\left(A \Phi_{\sigma}(G)\right) \approx \Psi_{\tau}\left(A \Phi_{\sigma}\left(G^{\prime}\right)\right) \\
& \rightarrow \Psi_{(\sigma) \tau}(A) G \approx \Psi_{(\sigma) \tau}(A) G^{\prime},
\end{aligned}
$$

where the next to last implication follows by the definition of s-MAJ ${ }^{\mathrm{E}}$.

It remains to verify (i)-(v).

Ad (i), let $F \in \mathrm{s}-\mathrm{MAJ}_{(\sigma) \tau}^{\mathrm{E}}$ and $G \in \mathrm{s}-\mathrm{MAJ}_{\sigma}^{\mathrm{E}}$. Then we have $\Phi_{(\sigma) \tau}(F) \Phi_{\sigma}(G)=$ $\Phi_{\tau}\left(F \Psi_{\sigma}\left(\Phi_{\sigma}(G)\right)\right)=\Phi_{\tau}\left(F G^{\prime}\right)$, with $G^{\prime}=\Psi_{\sigma}\left(\Phi_{\sigma}(G)\right) \approx G$ by IH. Since $F \in$ s-MAJ ${ }^{\mathrm{E}}$ we have $F \approx F$, so $F G \approx F G^{\prime}$ and so $\Phi_{\tau}(F G)=\Phi_{\tau}\left(F G^{\prime}\right)$ by IH. It follows that $\Phi_{(\sigma) \tau}(F) \Phi_{\sigma}(G)=\Phi_{\tau}(F G)$.

Ad (ii), let $A \in \mathfrak{M}_{(\sigma) \tau}$ and $B \in \mathfrak{M}_{\sigma}$; then we have

$$
\Psi_{(\sigma) \tau}(A) \Psi_{\sigma}(B)=\Psi_{\tau}\left(A \Phi_{\sigma}\left(\Psi_{\sigma}(B)\right)\right)=\Psi_{\tau}(A B)
$$

by the definition of $\Psi_{(\sigma) \tau}\left(\right.$ since $\left.\Psi_{\sigma}(B) \in \mathrm{s}-\mathrm{MAJ}^{\mathrm{E}} \subseteq \mathrm{s}-\mathrm{MAJ}\right)$ and by IH.

Ad (iii), let $F \in \mathrm{s}-\mathrm{MAJ}_{(\sigma) \tau}^{\mathrm{E}}$; then we have $F \approx F$ by the definition of s-MAJ${ }^{\mathrm{E}}$. By (i) we have

$$
\Psi_{(\sigma) \tau}\left(\Phi_{(\sigma) \tau}(F)\right) G=\Psi_{\tau}\left(\Phi_{(\sigma) \tau}(F) \Phi_{\sigma}(G)\right)=\Psi_{\tau}\left(\Phi_{\tau}(F G)\right)
$$


for all $G \in \mathrm{s}-\mathrm{MAJ}^{\mathrm{E}}$. Since $G \approx G^{\prime}$ implies $G, G^{\prime} \in \mathrm{s}-\mathrm{MAJ}^{\mathrm{E}}$ by Lemma 6.1 , we can derive by $\mathrm{IH}$

$$
\begin{aligned}
G \approx G^{\prime} \rightarrow F G \approx F G^{\prime} & \rightarrow \Phi_{\tau}(F G)=\Phi_{\tau}\left(F G^{\prime}\right) \\
& \rightarrow \Psi_{(\sigma) \tau}\left(\Phi_{(\sigma) \tau}(F)\right) G=\Psi_{\tau}\left(\Phi_{\tau}(F G)\right)=\Psi_{\tau}\left(\Phi_{\tau}\left(F G^{\prime}\right)\right) \approx F G^{\prime} .
\end{aligned}
$$

It follows that $\Psi_{(\sigma) \tau}\left(\Phi_{(\sigma) \tau}(F)\right) \approx F$.

Ad (iv), let $A \in \mathfrak{M}_{(\sigma) t}$; then by (ii) we have

$$
\Phi_{(\sigma) \tau}\left(\Psi_{(\sigma) \tau}(A)\right) B=\Phi_{\tau}\left(\Psi_{(\sigma) \tau}(A) \Psi_{\sigma}(B)\right)=\Phi_{\tau}\left(\Psi_{\tau}(A B)\right)=A B
$$

for all $B \in \mathfrak{M}_{\sigma}$. Since $\mathfrak{M}$ is extensional it follows that $\Phi_{(\sigma) \tau}\left(\Psi_{(\sigma) \tau}(A)\right)=A$.

$\operatorname{Ad}(\mathrm{v})$, let $F, F^{\prime} \in \mathrm{s}-\mathrm{MAJ}_{(\sigma) \tau}$ such that $F \approx F^{\prime}$. Let $B \in \mathfrak{M}_{\sigma}$; then $\Psi_{\sigma}(B) \in \mathrm{s}-\mathrm{MAJ}_{\sigma}^{\mathrm{E}}$, so $\Psi_{\sigma}(B) \approx \Psi_{\sigma}(B)$, and so $F \Psi_{\sigma}(B) \approx F^{\prime} \Psi_{\sigma}(B)$. Hence, by $\mathrm{IH}$,

$$
\Phi_{(\sigma) \tau}(F) B=\Phi_{\tau}\left(F \Psi_{\sigma}(B)\right)=\Phi_{\tau}\left(F^{\prime} \Psi_{\sigma}(B)\right)=\Phi_{(\sigma) \tau}\left(F^{\prime}\right) B
$$

for all $B \in \mathfrak{M}_{\sigma}$. Since in $\mathfrak{M}$ intensional and extensional equality coincide, it follows that $\Phi_{(\sigma) \tau}(F)=\Phi_{(\sigma) \tau}\left(F^{\prime}\right)$.

REMARK. It is essential that $\Phi$ is defined on s-MAJ instead of s-MAJ ${ }^{\mathrm{E}}$. The point is that for every $F \in \mathrm{s}-\mathrm{MAJ}^{\mathrm{E}}$ there exists an $F^{*} \in \mathrm{s}$-MAJ such that $F^{*}$ s-maj $F$, but we do not necessarily have $F^{*} \in \mathrm{s}-\mathrm{MAJ}$.

\$7. Majorizability and compactness in HEO. HEO is the typestructure of hereditarily effective operations, based on partial recursive application denoted by Kleene brackets, i.e. $\{x\} y=z$ iff $\exists k(T(x, y, k) \wedge U(k)=z)$ with Kleene's $T$-predicate and result-extracting function $U$. Moreover we use $\{x\} y \downarrow,\{x\} y \downarrow \leq n$, and $\{x\} y \uparrow$ to denote, respectively, $\exists k T(x, y, k), \exists k \leq n T(x, y, k)$, and $\forall k \neg T(x, y, k)$; in words, $\{x\} y$ converges (is defined), $\{x\} y$ converges in at most $n$ steps, and $\{x\} y$ diverges (is undefined). For the present section it suffices to define HEO for the types 0,1 and 2:

$$
\begin{aligned}
& \mathrm{HEO}_{0}=\mathbf{N} \\
& \mathrm{HEO}_{1}=\{x \mid \forall n\{x\} n \downarrow\}, x={ }_{1} x^{\prime} \text { iff } x, x^{\prime} \in \mathrm{HEO}_{1} \wedge \forall n\{x\} n=\left\{x^{\prime}\right\} n ; \\
& \mathrm{HEO}_{2}=\left\{y \mid \forall x \in \mathrm{HEO}_{1}\{y\} x \downarrow \wedge \forall x, x^{\prime}\left(x={ }_{1} x^{\prime} \rightarrow\{y\} x=\{y\} x^{\prime}\right)\right\} .
\end{aligned}
$$

When variables for functionals are taken to range over effective operations, the definitions of $\S 1$ of this paper yield notions of majorizability and compactness in HEO.

The main result of this paper, stated in $\S 5$, depends on:

$\mathfrak{U}$ is compact iff $\quad \exists F \forall A \in \mathfrak{U} F$ maj $A$ (resp. $F$ s-maj $A, A \leq F$ ).

Even if $\mathfrak{Q}$ consists of recursive objects it is not very likely that $F$ is a recursive object. This idea leads to the following negative result in HEO.

THEOREM. There exists an effective operation $y \in \mathrm{HEO}_{2}$ such that $y$ maj $y$ (resp. $y$ $\mathrm{s}$-maj $y, y \leq y$ in the sense of $\omega$-maj), but $y$ is not compact.

ProOF. Let $\Phi$ be any partial recursive function that cannot be extended to a total recursive one (e.g. $\Phi=\lambda i$. $\{i\} i)$. Define an index $y$ by

$$
\{y\} x \simeq \mu n\left[\exists i \leq n\left(\Phi_{i \downarrow} \leq n \wedge \neg \Phi i \downarrow \leq\{x\} i\right)\right],
$$

where $\Phi i \downarrow^{\leq k}$ means that $\Phi i$ converges in at most $k$ steps. By our assumption on $\Phi$ it follows that $\{y\} x \downarrow$ for every $x \in \mathrm{HEO}_{1}$ (i.e. $x$ is the index of a total recursive 
function). For, $\{y\} x \uparrow$ implies $\forall i(\Phi i \uparrow \vee \Phi i \downarrow \leq\{x\} i)$ and so $\Psi$ defined by $\Psi_{i}=\Phi i$ if $\Phi i \downarrow \leq\{x\} i$, and 0 else, would be a total recursive extension of $\Phi$. Moreover $x={ }_{1} x^{\prime}$ implies $\{y\} x=\{y\} x^{\prime}$. It follows that $y \in \mathrm{HEO}_{2}$. For all $x, x^{*} \in \mathrm{HEO}_{1}$ we have that $x^{*}$ maj $x$ (resp. $x^{*}$ s-maj $x, x \leq x^{*}$ in the sense of $\omega$-maj) implies $\forall i\left\{x^{*}\right\} i \geq\{x\} i$ and hence $\{y\} x^{*} \geq\{y\} x$. It follows that $y$ maj $y$ (resp. $y$ s-maj $y, y \leq y$ in the sense of $\omega$-maj). However, $y$ is not compact, since $y$ is not bounded on the compact set

$$
\mathbb{C}=\left\{x \in \mathrm{HEO}_{1} \mid \forall i\left(\Phi i \downarrow \rightarrow\{x\} i \leq \mu n\left[i \leq n \wedge \Phi_{i \downarrow} \leq n\right] \text { and } \Phi i \uparrow \rightarrow\{x\} i=0\right)\right\} \text {. }
$$

§8. Relative compactness. In this section we study the nature of COMPACT (see $\S 1)$ as (intensional) sub-typestructure of the full typestructure. To this end we introduce the following notion of reducibility: $F$ is compact in $G$ iff $F$ is definable in $G$ by compact functionals.

THEOREM. Every functional is compact in any noncompact functional.

Proof. Let $A \in\left(\sigma_{1}\right) \cdots\left(\sigma_{k}\right) 0$ be an arbitrary functional of the full typestructure and let $N$ be a noncompact functional, say of type $(\rho)(\tau) 0$ (other cases are similar). Then there exist compact sets $\mathfrak{B} \subset \rho$ and $\mathbb{C} \subset \tau$ such that $N \mathfrak{B C}$ is not finite; hence for every $n \in \mathbf{N}$ there exist $B_{n} \in \mathfrak{B}$ and $C_{n} \in \mathbb{C}$ such that $N B_{n} C_{n} \geq n$. We may assume that all pairs $\left(B_{n}, C_{n}\right)$ are different. Now consider the functional $M$ of type $((\rho)(\tau) 0)(\rho)(\tau) 0$ defined by $M F X Y=\min (F X Y, n)$ if $X=B_{n} \wedge Y=C_{n}, M F X Y=0$ otherwise. Then $M$ is clearly compact since $M F X Y \leq F X Y$ for all $F, X, Y$. Moreover $M N B_{n} C_{n}$ $=n$ for all $n \in \mathbf{N}$. Now consider the functionals $B_{A} \in\left(\sigma_{1}\right) \cdots\left(\sigma_{k}\right) \rho$ and $C_{A} \in\left(\sigma_{1}\right) \cdots\left(\sigma_{k}\right) \tau$ defined by $B_{A} \vec{Z}=B_{A} \vec{Z}$ and $C_{A} \vec{Z}=C_{A \vec{Z}}$ for all $\vec{Z}$. $B_{A}$ and $C_{A}$ are compact since $\mathfrak{B}$ and $\mathfrak{C}$ are compact. Since $A=\lambda \vec{Z} . M N\left(B_{A} \vec{Z}\right)\left(C_{A} \vec{Z}\right)$ it follows that $A$ is compact in $N$.

COROLLARY. COMPACT (and by $\S 5$ also MAJ, s-MAJ and $\omega$-MAJ) is maximal in the sense that adding one arbitrary noncompact functional and closing under application yields the full typestructure.

\section{REFERENCES}

[B1] M. BEZEM, Isomorphisms between HEO and $\mathrm{HRO}^{\mathrm{E}}, \mathrm{ECF}$ and $\mathrm{ICE}^{\mathrm{E}}$, this JOURNAL, vol. 50 (1985), pp. 359-371.

[B2] - Strongly majorizable functionals of finite type: a model for barrecursion containing discontinuous functionals, this JoURNAL, vol. 50 (1985), pp. 652-660.

$[\mathrm{K}] \mathrm{U}$. KOHLENBACH, Majorisierbare Funktionale und rekursionstheoretische Modelle für $W$. Friedrichs Funktionalkalküle, Diplomarbeit, J. W. Goethe Universität, Frankfurt, 1986.

[T] A. S. TROELSTRA (editor), Metamathematical investigation of intuitionistic arithmetic and analysis, Lecture Notes in Mathematics, vol. 344, Springer-Verlag, Berlin, 1973.

$[\mathrm{Z}] \mathrm{Z}$. ZUCKER, Proof theoretic studies of systems of iterative inductive definitions and subsystems of analysis, Ph.D. thesis, Stanford University, Stanford, California, 1971.

CENTRE FOR MATHEMATICS AND COMPUTER SCIENCE

P.O. BOX 4079

1009 AB AMSTERDAM, THE NETHERLANDS 